\title{
Nutrition en réanimation : un pas en avant, deux pas en arrière !
}

\section{Nutrition in critically ill patients: one step up and two steps back!}

\author{
B. Raynard $\cdot$ J.-C. Preiser \\ (C) SRLF et Springer-Verlag France 2012
}

Qu'avons-nous appris depuis 20 ans ? Que nous ont appris les quelques 2000 articles publiés sur le sujet depuis dix ans (requête Pubmed «nutrition AND intensive care ») ? Probablement d'abord, comme souvent, qu'il ne faut pas mettre la charrue avant les bœufs. Que peut-être l'énergie dépensée par beaucoup d'entre nous à la promotion de la pharmaconutrition, avant même que nous nous mettions d'accord sur le bon nombre de calories et la bonne voie d'administration au bon moment, n'avait pas été la meilleure façon d'optimiser la prise en charge nutritionnelle du patient de réanimation. La promotion de la pharmaconutrition a parfois été plus le résultat d'une politique commerciale que d'une démarche scientifique rigoureuse.

L'émotion suscitée par la publication, en 2011, d'un travail réalisé dans trois unités de soins intensifs belges (4640 patients randomisés), en est peut-être un parfait exemple [1]. Voilà qu'en pleine réflexion sur l'optimisation de l'apport calorique et sur la limitation du déficit énergétique des malades de réanimation, un nouveau pavé belge est jeté dans la mare. Une nutrition parentérale de complément administrée précocement (conformément aux recommandations européennes) n'améliorerait pas le pronostic en réanimation comparée à une nutrition parentérale de complément tardive, instaurée après le septième jour de nutrition entérale insuffisante (stratégie recommandée par les Nord-Américains). Le déficit calorique cumulé dans le groupe « parentérale tardive » était proche de 10000 kilocalories au terme des sept premiers jours de réanimation, et contre toute attente, dans ce groupe, le taux de complications infectieuses et la durée de séjour en réanimation étaient significativement moins importants que dans le groupe « parentérale précoce », contredisant ainsi certains travaux rétrospectifs plus anciens.

\footnotetext{
B. Raynard $(\bowtie)$

Service de réanimation-USCM, institut Gustave Roussy, Villejuif, France

e-mail : raynard@igr.fr

J.-C. Preiser

Département des soins intensifs, hôpital Erasme,

Bruxelles, Belgique
}

Rappelons-nous encore que l'étude EDEN, comparant nutrition entérale à débit d'emblée élevé versus débit progressivement augmenté en fonction de la tolérance digestive chez des patients atteints de syndrome de détresse respiratoire aigu (SDRA) ne montrait aucune différence de morbimortalité entre les deux groupes [2]. Là encore le déficit calorique cumulé du groupe «progressif » atteignait 8000 kcalories à J6. Une autre étude prospective, malheureusement non encore publiée pourrait apporter un son de cloche différent sur ce sujet [3]. En effet, une équipe suisse a démontré le bénéfice de la complémentarité entérale-parentérale chez un sous-groupe de patients (305 patients, soit $15 \%$ des admissions des deux centres concernés) pour lesquels la nutrition entérale optimisée était incapable d'apporter plus de $60 \%$ des besoins caloriques au troisième jour de réanimation. Mais finalement, les études belges et suisses s'opposentelles ? Probablement pas, puisqu'elles portent le même message - la nutrition entérale doit être la voie d'administration de première intention en l'absence de contre-indication et doit être optimisée avant d'envisager une nutrition parentérale de complément. Même si la vérité appartient souvent à celui qui parle le plus fort et le dernier, espérons que de ces données, à première vue contradictoires, naisse un message unique, simple et de bon sens !

Et les pharmaconutriments nous direz-vous, que deviennent-ils ? Leur place reste incertaine ou en tout cas instable. Après la publication de trois essais contrôlés randomisés montrant un bénéfice de survie d'une nutrition entérale enrichie en acides gras n-3 dans le SDRA, deux essais, l'un espagnol l'autre nord-américain, nous disent maintenant le contraire [4,5]. La glutamine a-t-elle plus d'avenir ? Nombreux sont ceux qui le croient. L'étude française publiée en 2006 leur avait redonné espoir, mais voilà que trois nouvelles publications, toutes les trois négatives, devraient mettre à mal ces croyances [6-8]. Même si les recommandations, française et internationale, continuent de soutenir l'indication de la glutamine, de façon assez large, en réanimation pour les patients recevant une nutrition parentérale a priori exclusive, les résultats de ces dernières études devraient modérer l'enthousiasme de ces recommandations. Et que dire de l'arginine, tellement adulée pour ces bienfaits 
périopératoires et tant décriée pour ses effets délétères au cours des sepsis sévères et des chocs septiques. Dans ce numéro de la revue Réanimation, Michel Hasselmann nous proposera une vision actualisée et raisonnée des avantages et inconvénients de l'arginine [9].

Voilà donc dans quel état d'incertitude se trouve la nutrition en réanimation. Mais ceci n'est pas un prétexte pour ne pas faire. Nous avons la certitude que la nutrition, bien faite, peut apporter un bénéfice de morbimortalité en réanimation. Fondée sur les recommandations longuement réfléchies et discutées, souvent critiquables, la prise en charge nutritionnelle se doit d'être simple, adaptée à chaque patient, et surtout régulièrement réévaluée afin de dépister des effets indésirables méconnus, comme pour toute intervention thérapeutique.

Antoine de Saint-Exupéry disait que « la vérité de demain se nourrit de l'erreur d'hier ». Souhaitons-nous alors, aujourd'hui, quelques erreurs pour aboutir, dès demain, à une vérité plus fiable et compréhensible par tous concernant la nutrition en réanimation!

\section{Références}

1. Casaer MP, Mesotten D, Hermans G, et al (2011) Early versus late parenteral nutrition in critically ill adults. New Engl J Med 365:506-17
2. National Heart, Lung, and Blood Institute Acute Respiratory Distress Syndrome (ARDS) Clinical Trials Network, Rice TW, Wheeler AP, et al (2012) Initial trophic vs full enteral feeding in patients with acute lung injury: the EDEN randomized trial. JAMA 307:795-803

3. Thibault R, Graf S, Heidegger CP, et al (2011) Nutrition parentérale complémentaire aux soins intensifs pour une couverture énergétique optimale : évolution clinique améliorée. Nutr Clin Métabol 25(suppl 2):S40

4. Grau-Carmona T, Morán-García V, García-de-Lorenzo A, et al (2011). Effect of an enteral diet enriched with eicosapentaenoic acid, gamma-linolenic acid and anti-oxidants on the outcome of mechanically ventilated, critically ill, septic patients. Clin Nutr 30:578-84

5. Rice TW, Wheeler AP, Thompson BT, et al (2011) Enteral Omega3 Fatty Acid, $\gamma$-Linolenic Acid, and Antioxidant Supplementation in Acute Lung Injury. JAMA 306:1574-81

6. Andrews PJ, Avenell A, Noble DW, et al (2011) Randomised trial of glutamine, selenium, or both, to supplement parenteral nutrition for critically ill patients. BMJ 342:d1542

7. Wernerman J, Kirketeig T, Andersson B, et al (2011) Scandinavian glutamine trial: a pragmatic multi-centre randomised clinical trial of intensive care unit patients. Acta Anesthesiol Scand 55:812-8

8. Grau T, Bonet A, Miñambres E, et al (2011) The effect of L-alanylL-glutamine dipeptide supplemented total parenteral nutrition on infectious morbidity and insulin sensitivity in critically ill patients. Crit Care Med 39:1263-8

9. Boisramé-Helms J, Meyer G, Meziani F, Hasselmann M (2012) Implications et intérêt potentiel de l'arginine comme pharmaconutriment chez le malade de réanimation. Réanimation vol. 21 [In press] 